\title{
Excess maternal salt or fructose intake programmes sex-specific, stress- and fructose-sensitive hypertension in the offspring
}

\author{
Clint Gray ${ }^{1,2}$, Sheila M. Gardiner ${ }^{3}$, Matthew Elmes ${ }^{4}$ and David S. Gardner ${ }^{1 *}$ \\ ${ }^{1}$ School of Veterinary Medicine and Science, University of Nottingham, Sutton Bonington Campus, Sutton Bonington, \\ Leicestershire LE12 5RD, UK \\ ${ }^{2}$ The Liggins Institute, University of Auckland, Auckland, New Zealand \\ ${ }^{3}$ School of Medicine, University of Nottingham, Queens Medical Centre, Nottingham NG7 2UH, UK \\ ${ }^{4}$ School of Biosciences, University of Nottingham, Sutton Bonington Campus, Sutton Bonington, Leicestershire LE12 5RD, UK \\ (Submitted 17 July 2015 - Final revision received 14 October 2015 - Accepted 3 November 2015 - First published online 10 December 2015)
}

\section{Abstract}

The Western diet is typically high in salt and fructose, which have pressor activity. Maternal diet can affect offspring blood pressure, but the extent to which maternal intake of excess salt and fructose may influence cardiovascular function of the offspring is unknown. We sought to determine the effect of moderate maternal dietary intake of salt and/or fructose on resting and stimulated cardiovascular function of the adult male and female offspring. Pregnant rats were fed purified diets $( \pm 4 \%$ salt $)$ and water $( \pm 10 \%$ fructose $)$ before and during gestation and through lactation. Male and female offspring were weaned onto standard laboratory chow. From 9 to 14 weeks of age, cardiovascular parameters (basal, circadian and stimulated) were assessed continuously by radiotelemetry. Maternal salt intake rendered opposite-sex siblings with a $25-\mathrm{mmHg}$ difference in blood pressure as adults; male offspring were hypertensive (15 mmHg mean arterial pressure (MAP)) and female offspring were hypotensive $(10 \mathrm{mmHg}$ MAP) above and below controls, respectively. Sex differences were unrelated to endothelial nitric oxide activity in vivo, but isolation-induced anxiety revealed a significantly steeper coupling between blood pressure and heart rate in salt-exposed male offspring but not in female offspring. MAP of all offspring was refractory to salt loading but sensitive to subsequent dietary fructose, an effect exacerbated in female offspring from fructose-fed dams. Circadian analyses of pressure in all offspring revealed higher mean set-point for heart rate and relative non-dipping of nocturnal pressure. In conclusion, increased salt and fructose in the maternal diet has lasting effects on offspring cardiovascular function that is sex-dependent and related to the offspring's stress-response axis

Key words: Rats: Hypertension: Fructose: Salt: Maternal nutrition: Stress

Ancestral man is predicted to have eaten a diet high in fibre, $\mathrm{K}$, complex carbohydrates and protein and low in $\mathrm{Na}$, refined sugars and energy density. Typically, a palaeolithic diet provided a plant-to-animal energy ratio of $1: 1$, with the net acid-load being alkaline $^{(1,2)}$. Analyses of the diets of modern hunter-gatherer populations support these predictions ${ }^{(2,3)}$. Since this time, when physiological and metabolic systems were evolving, there has been a gradual transition away from this palaeolithic diet. With the emergence of agriculture (about 7-5000 years ago) through to the industrial revolution (about last 100 years), the 'modern diet' has rapidly become low in fibre and high in $\mathrm{Na}$, simple sugars and energy density ${ }^{(4)}$. When superimposed on the palaeolithic genotype and physiology, the modern diet has resulted in an increased incidence of non-communicable diseases (NCD), which is estimated to account for $60 \%$ of all deaths worldwide $^{(5)}$. The economic impact of NCD is vast: $\$ 558, \$ 237$ and $\$ 33$ billion in China, India and the UK, respectively ${ }^{(6)}$, whereas $\$ 750$ billion is spent annually in the USA for diabetes and hypertension alone ${ }^{(7)}$.

Modification of diet offers an achievable and economically beneficial prevention strategy for NCD. Short-term consumption of a 'palaeolithic' diet produces significant reductions in blood pressure, cholesterol, TAG and insulin resistance ${ }^{(8)}$. In addition, reduced salt intake (e.g. $3 \mathrm{~g} / \mathrm{d}$ ) is predicted to reduce all-cause mortality in the USA by 44-92000 individuals, saving an estimated \$10-24 billion annually ${ }^{(9)}$. Reducing sugar-sweetened beverage consumption by 1 serving/d reduced systolic blood pressure by $1.8 \mathrm{mmHg}^{(10)}$. Earlier dietary intervention, for example to pregnant mothers or those considering pregnancy, may have added benefit, as an adverse periconceptional and/or prenatal nutritional exposure has been shown to increase the risk of NCD (e.g. CVD or metabolic disease) in the adult

Abbreviations: $\mathrm{CD}$, control diet; FD, fructose diet; L-NAME, $N^{\mathrm{G}}$-nitro-L-arginine methyl ester; NCD, non-communicable disease; SD, salt diet.

* Corresponding author: Dr D. S. Gardner, fax +44·115.951·6415, email david.gardner@nottingham.ac.uk 
offspring $^{(11-13)}$ - a paradigm referred to as the developmental programming of health and disease.

The majority of developmental programming studies to recapitulate either a 'Westernised' or under/over-nourished diet in experimental models have used a low-protein or a high-fat and/or a high-sugar paradigm ${ }^{(14-16)}$. In the UK, although higher than optimal (reference nutrient intake) intake of SFA is observed, high total fat intake is not. Indeed, data from the National Diet and Nutrition Survey suggest that total fat consumption is close to recommended but that fructose and salt intake remain high ${ }^{(17)}$. In the USA, a similar dietary pattern of high fructose and high salt intake has been observed, raising concerns about increased CVD risk ${ }^{(18,19)}$.

The delayed programming effect of a maternal diet high in simple sugars (e.g. fructose ${ }^{(20)}$ ) or salt has been considered ${ }^{(21,22)}$. Feeding sucrose to pregnant rats can influence hepatic metabolism and reduce offspring birth weight ${ }^{(23)}$, and fructose-feeding during lactation renders the resultant adult offspring vulnerable to cardiometabolic risk ${ }^{(20)}$. A maternal diet high in salt is one of the few dietary challenges to repeatedly produce hypertensive offspring $^{(21,24)}$. More importantly, increased intake of salt in (or added to) food potentiates intake of simple sugars (e.g. from drinking sugar-sweetened beverages $)^{(25)}$. As each is known to influence cardiovascular health, it is important to consider their potential interaction experimentally. Sex-specific effects are widely observed in developmental programming studies ${ }^{(26)}$; sex is an important consideration with regard to disease susceptibility, ${ }^{(27)}$ and there has been recent criticism of sex bias (in favour of male offspring) in translational medicine studies $^{(28,29)}$. It is therefore important to also consider potential sex-specific responses after maternal dietary intervention with respect to offspring cardiovascular function.

To date, no study has considered the delayed cardiovascular consequences on adult offspring (male and female) of the combined intake of fructose and salt by the dam. Excess salt in the diet increases fluid intake; disappointingly, this tends to be of sugarsweetened beverages ${ }^{(25)}$. We anticipate that high maternal intake of fructose and salt renders adult offspring prone to hypertension and hypersensitive to further consumption of salt or fructose. The aim of the present study was to characterise the cardiovascular health of adult male and female rat offspring after maternal consumption of a high salt diet (SD) and/or fructose diet (FD) before and during her pregnancy and for the duration of her lactation. Baseline cardiovascular health of all offspring was assessed 24/7 by radiotelemetry, as previously described by us after maternal salt intake ${ }^{(24)}$. Cardiovascular hypersensitivity in vivo was assessed during four further experimental studies: (1) during sympathetic activation induced by anxiety-related isolation, (2) during nitric oxide blockade with $N^{\mathrm{G}}$-nitro-L-arginine methyl ester (L-NAME), (3) during dietary salt loading or (4) dietary fructose loading to determine whether postnatal response is conditioned by prenatal exposure. During each challenge, all data recorded were submitted for further non-linear regression analyses to determine potential effects on cardiovascular function through the circadian cycle. Finally, offspring hearts were studied ex vivo using the perfused Langendorff system to assess isolated cardiac function. For all outcome measures, we have assessed cardiovascular responses in different-sex siblings.

\section{Methods}

\section{Ethics}

Animal procedures were carried out under license and in accordance with the Home Office animals (Scientific Procedures) Act 1986 and approved by the local animal welfare and ethical review board of the University of Nottingham.

\section{Diet design}

In brief, Sprague-Dawley dams (190-200 g; 8-10 weeks of age) were kept in a temperature $\left(20-22^{\circ} \mathrm{C}\right)$ - and humidity (55-65\%)controlled environment and subjected to a 12-h light/dark cycle (07.00-19.00 h). Rats were randomly assigned to one of four treatment (diet) groups: (1) control diet (CD; $n$ 6), fed purified standard chow (TD.08164; Teklad Harlan) and tap water; (2) SD $(n$ 6), fed purified standard chow with $4 \% \mathrm{NaCl}$ added (TD.08162; Teklad Harlan) and tap water; (3) FD ( $n$ 6), fed purified standard chow (TD.08164) and tap water with $10 \%$ fructose (Sigma-Aldrich) added; (4) fructose/salt diet (FSD; $n$ 6), fed purified SD (TD.08162) and tap water with $10 \%$ fructose added. Diet composition has been published previously ${ }^{(30)}$. All rats were fed the experimental diets ad libitum for at least $28 \mathrm{~d}$ before conception and throughout gestation and lactation.

\section{Radiotelemetry and baseline cardiovascular recording}

At 9 weeks of age, one male and one female offspring from each litter were surgically instrumented for radiotelemetric recording of blood pressure (TA11PA-C40; DSI) from the descending abdominal aorta, as described previously ${ }^{(31)}$. In brief, the rats were fully anaesthetised (fentanyl citrate; Sublimaze, Janssen-Cilag and medetomidine hydrochloride; Domitor, Pfizer; $300 \mu \mathrm{g} / \mathrm{kg}$ of each i.p.) for probe implantation (TA11PA-C40; DSI). Anaesthesia was reversed (Antisedan, Pfizer; $1 \mathrm{mg} / \mathrm{kg}$ ) and analgesia was administered (buprenorphine; Buprecare, Animalcare; $0.02 \mathrm{mg} / \mathrm{kg}$ s.c.) together with a long-acting antibiotic (Amoxycare LA; $0.05 \mathrm{ml}$ i.m.). All twentyfour rats that underwent surgery completed the study, and all were subsequently housed with a same-sex sibling to minimise stress. Cardiovascular variables were recorded (Dataquest GOLD version 4.02; DSI) at intervals $(15 \times 2 \mathrm{~s}$ periods per $15 \mathrm{~min}$ ) during a 5-7-d recovery and baseline period and during cardiovascular challenges, which each lasted for further 5-7-d periods. Male and female siblings were recorded simultaneously, each with a same-sex cage mate present at all times, but challenges were conducted in a random order. At the end of all experiments, rats were euthanised in a sealed chamber using a rising concentration of $\mathrm{CO}_{2}$, followed by cervical dislocation after confirmation of cardiac arrest.

\section{Radiotelemetry and stimulated cardiovascular recording}

$\mathrm{CV}$ challenge 1: Isolation-induced anxiety - after a recovery period, the untelemetered sibling was removed from the cage for a 24-h period, and blood pressure and heart rate were recorded continuously (i.e. $\times 215 \mathrm{~s}$ periods/min; 2880 data points in total). Thereafter, siblings were reunited and recording 
continued at intervals. With 5-7-d recovery and wash-out periods between each challenge, telemetered rats were subjected to three further experimental studies in a randomised manner, each lasting $5 \mathrm{~d}$ with a further $2 \mathrm{~d}$ of recording during recovery; CV challenge 2: Nitric oxide blockade - the drinking water was substituted for fresh water with L-NAME dissolved at a concentration of $150 \mu \mathrm{g} / \mathrm{ml}$ (equivalent to $4.1 \mathrm{mg} \mathrm{L-NAME} / \mathrm{d}$ ); CV challenge 3: Salt loading - standard chow was substituted for purified chow with $4 \% \mathrm{NaCl}$ (TD.08162); and CV challenge 4 : Fructose loading - the drinking water was substituted for fresh water with $10 \%$ fructose solution.

\section{The isolated heart (Langendorff) preparation}

One male and one female offspring from each control or salt-exposed dams (offspring of fructose-fed dams were not included) were randomly selected, anaesthetised (3\% isofluorane in 2 litres $/ \mathrm{min} \mathrm{O}_{2}$ ) and killed by cervical dislocation. Within $90 \mathrm{~s}$, the heart was excised and cannulated via the aorta to Langendorff perfusion apparatus (AD Instruments) and reverse-perfused with Krebs Henseleit buffer (118 mм-NaCl, $4.7 \mathrm{~mm}-\mathrm{KCl}, \quad 1.2 \mathrm{~mm}-\mathrm{KH}_{2} \mathrm{PO}_{4}, 1.2 \mathrm{~mm}-\mathrm{MgSO}_{4}, 25 \mathrm{~mm}-\mathrm{NaHCO}_{3}$, $11 \mathrm{~mm}$-glucose and $1.25 \mathrm{~mm}^{-\mathrm{CaCl}_{2}}, \mathrm{pH} 7.4$, bubbled with $\left.95 \% / 5 \% \mathrm{O}_{2} / \mathrm{CO}_{2}\right)$. Perfusion was maintained at a constant pressure of $60 \mathrm{mmHg}$, with the perfusate warmed to $37 \cdot 4^{\circ} \mathrm{C}$ and the heart immersed in a water-jacketed temperature-controlled glass chamber set at $37 \cdot 4^{\circ} \mathrm{C}$, therefore ensuring normothermia throughout the perfusion protocol. Contractile function (left ventricular developed pressure) was determined by an intravascular balloon, adjusted to an end diastolic pressure of 5-10 mmHg. Data were recorded for a 30-min baseline period after 15-30 min stabilisation via transducers (Senso-Nor 844; $\mathrm{AD}$ Instruments) using the Powerlab Acquisition System (AD Instruments).

\section{Statistics}

The study was designed with a 2 ( \pm fructose $) \times 2( \pm$ salt $)$ factorial structure and was analysed by a general linear model (GLM) approach for normally distributed data or after log transformation for a skewed error distribution (GenStat version 16; VSNi). All data are presented as mean values with their standard errors or standard error of the difference between means (for a more conservative estimate of the contrast variance). Although $P \leq 0.050$ was accepted as indicating statistical significance, values of $P$ from 0.06 to 0.09 are also presented to indicate effects falling close to the arbitrary significance boundaries. Using one male or female offspring per litter per determination avoids complicating the statistical model with shared intra-litter variance. For offspring cardiovascular analyses, data were either tested as summary measures (e.g. hourly means of blood pressure) or, for circadian analyses, by incorporating all recorded cardiovascular data (e.g. 2880 datapoints per animal; 14 400-17 280 data points per group ( $n$ 5-6 animals of each sex) into a non-linear regression model fitting a Fourier curve $(Y=\alpha+\beta \sin (2 \pi(X+\varepsilon) / w))$ to derive four parameters $\alpha$, set-point; $\beta$, amplitude; $w$, wavelength; and $\varepsilon$, offset - which were analysed by GLM.

\section{Results}

\section{Maternal food intake}

At conception, food intake was similar in rats fed SD but marginally reduced in those with fructose-sweetened water available (CD, 10.3 (sem 1.0) g/d; SD, 10.9 (sem 0.9) g/d; FD, 9.36 $($ SEM $0 \cdot 8) \mathrm{g} / \mathrm{d} ;$ FSD, $7 \cdot 02($ SEM 0.9$\left.) \mathrm{g} / \mathrm{d} ; P_{\text {fructose }}=0 \cdot 01\right)$. Food intake increased with advancing gestational age: by day 20 of gestation (term approximately day 21), rats were eating approximately double the quantity at conception, and those rats with fructose-sweetened water available were still consuming marginally less food (CD, $22.6(\operatorname{sem} 2 \cdot 2) \mathrm{g} / \mathrm{d}$; SD, $21.8($ SEM $2 \cdot 0) \mathrm{g} / \mathrm{d}$; FD, $18.2($ SEM 1.9$) \mathrm{g} / \mathrm{d} ;$ FSD, $\left.16.6(\operatorname{sem} 1.9) \mathrm{g} / \mathrm{d} ; P_{\text {fructose }}=0.02\right)$. Nevertheless, using the AIN-93G formulation and despite a marginal reduction in food intake in those rats with fructose available, the diets (TD.08164 and TD.08162) still met macronutrient and micronutrient requirements for pregnant rats ${ }^{(32)}$.

\section{Resting cardiovascular status of adult offspring}

Prenatal exposure to SD significantly increased blood pressure in male offspring, with systolic, mean and diastolic pressures being $15 \mathrm{mmHg}$ higher than age-matched CD (Table 1; Fig. 1(a)). In contrast, female siblings tended to be hypotensive, with systolic, mean and diastolic pressures being $10 \mathrm{mmHg}$ lower than dietary controls (Table 1; Fig. 1(b)). Circadian analyses of pressure and heart rate, incorporating all measured data points for each animal within each diet group, suggested less dipping of nocturnal heart rate in male offspring exposed in utero to high maternal salt (Fig. 1(c)) and in female offspring exposed in utero to high maternal fructose (Fig. 1(d)). The latter, additionally, exhibited less dipping of nocturnal blood pressure (Fig. 1(e)). Such effects, despite the absence of excessive dietary intake postnatally, suggest long-term programming of cardiovascular sensitivity and reactivity in the offspring. We then tested this hypothesis in a number of experiments:

\section{Stimulated cardiovascular responses: isolation-induced stress}

Immediately upon removal of their sibling from the cage, the single-housed telemetered offspring exhibited a robust cardiovascular response (Fig. 2(a)-(d)). Despite differing baselines, the magnitude of the change in pressure and heart rate was similar between dietary groups, but when the slopes of the relationship between paired values were analysed, the male, but not female, offspring of dams fed salt diet exhibited a significantly steeper response; the calculated slopes for male offspring were CD, 3.26 (95\% CI 3.02, 3.49); FD, 2.81 (95\% CI $2.63,2.99)$ ) SD, 5.36 (95\% CI 5.17, 5.55); FSD, 5.38 (95\% CI $5 \cdot 15,5.60)$ beats/min per $\mathrm{mmHg} ; P<0.001$, and for female offspring the slopes were CD, 4.77 (95\% CI 4.59, 5.08); FD, 4.26 (95\% CI 3.91, 4.60); SD, 4.47 (95\% CI 4.25, 4.69); FSD, 3.30 (95\% CI 3.12, 3.48) beats/min per mmHg (Fig. 2(e) and (f)). In short, the male offspring of dams fed a high-SD are hypertensive, with greater short-term cardiovascular reactivity to anxiety-related stimuli that leads on, in the long term, to less 
Table 1. Summary measures analysis of resting cardiovascular status of adult male and female offspring from dams consuming salt and/or fructose

(Mean values with their standard errors of the difference between means)

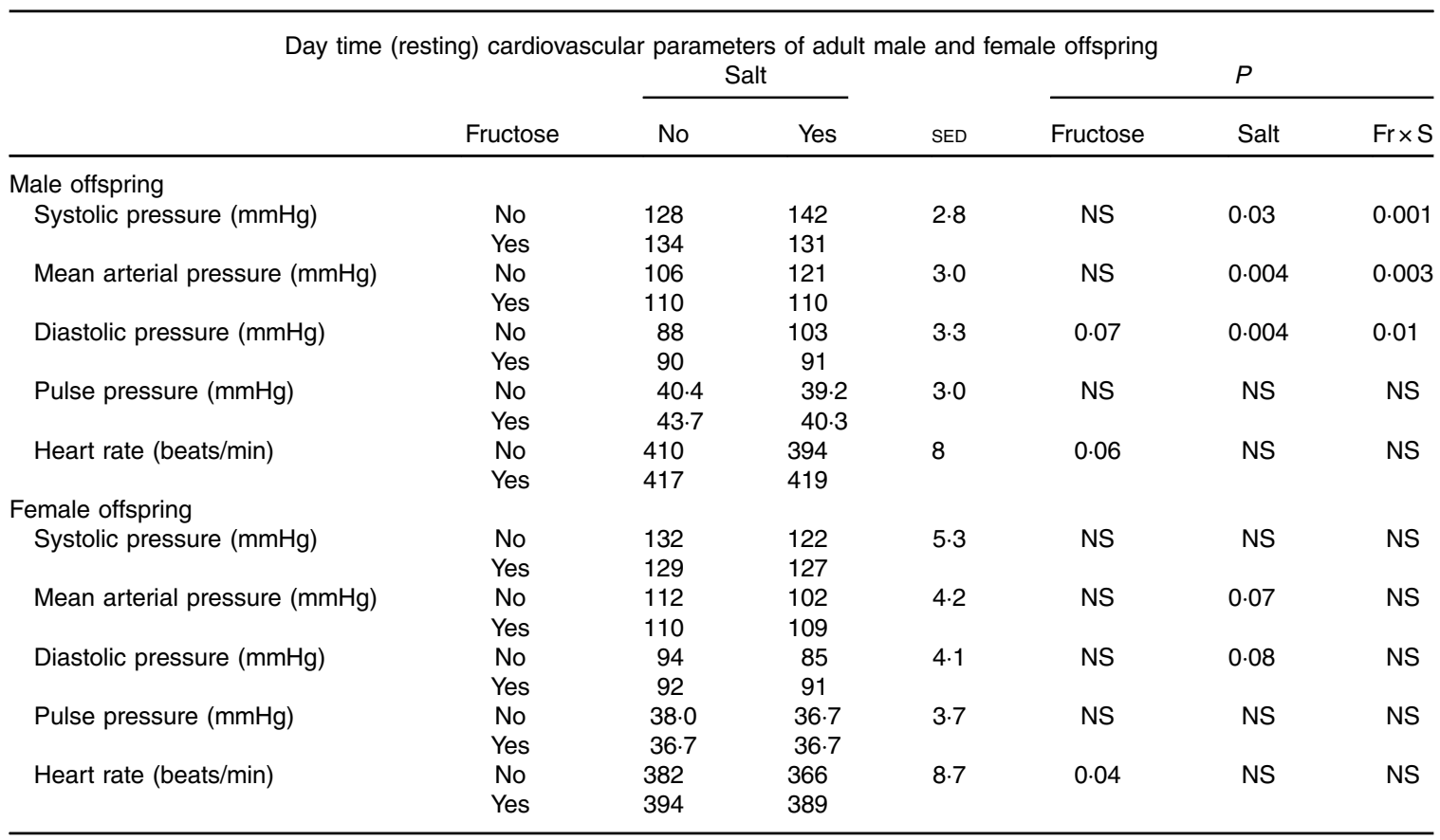

$\mathrm{Fr} \times \mathrm{S}$, interaction of fructose $\times$ salt.

Blood pressures and heart rate were derived from radiotelemetric signals and reflect average values during the 'resting' period (i.e. day time; 07.00 to 20.00 hours) over a 7-d period. Mean values for the comparison from $n$ 5-6 male or female offspring per dietary group ( $n$ 5-6 dams per dietary group). Data were analysed by 2 (salt, yes/no) $\times 2$ (fructose, yes/no) factorial ANOVA within each sex (GenStat version 13). Statistical significance was accepted at $P<0.05$

dipping of heart rate at night. We then assessed whether such a phenotype was underpinned by programmed cardiovascular changes in (a) the periphery, by examining cardiovascular function on a background of tonic endothelial nitric oxide blockade, and in (b) the heart, by using the Langendorff technique in isolated hearts.

Stimulated cardiovascular responses: nitric oxide blockade. Upon consumption of L-NAME, the mean arterial pressure increased significantly in both sexes of all groups (Fig. 3(a) and (b)), with the magnitude of change (i.e. increase from baseline) being similar between groups and sexes pooled estimate, 43.3 (SEM $2.6 \mathrm{mmHg}$ ). The oscillation in heart rate increased with the duration of L-NAME treatment in both male and female offspring i.e. the $\beta$-coefficient increased from 37.1 (SEM 3.1) beats/min (days 1-2) to 50.1 (SEM 3.0) beats/min (days 4-5) for male and female offspring alike (Fig. 3(c) and (d)). Despite L-NAME treatment, circadian analyses indicated heart rate to remain elevated in male, but not female, offspring of salt-fed dams (352 v. 337 (sEM 2.1) beats/min; $P<0.001$; Fig. 3(e)). In addition, the reduced dipping of heart rate at night in the male offspring from salt-loaded dams was retained (Fig. 3(e)). Similarly, adult female, but not male, offspring of fructose-fed dams retained higher average heart rates: 384 v. 362 (SEM 2.1) beats/min (Fig. 3(f)). Programmed sex-specific pathways in the adult offspring, which independently influence adult cardiovascular control after maternal salt or fructose loading, were therefore beginning to emerge: for male offspring, maternal
high-SD renders them reactive to further cardiovascular stressors as adults; for female offspring, maternal high fructose has a similar effect. Each was apparently independent of endothelial NOx status.

Adult offspring isolated heart function at 8 weeks of age. With hearts mounted on the Langendorff apparatus, heart rate was higher $(P=<0 \cdot 001)$ in the female offspring of dams fed SD (male, $312 v$. 308; female, $310 v .330$ beats/min for CD v. SD, respectively) but left ventricular developed pressure (male, median 39 (IQR 17-45) v. 39 (IQR 35-53); female, 51 (IQR 46-56) v. 48 (IQR 39-50) $\mathrm{mmHg}$ for $\mathrm{CD} v$. SD, respectively) and the maximal positive derivative of the rate of change in developed pressure $(+d p / d t)$ were not different between groups (male offspring, median 1076 (IQR 609-1449) v. 1617 (IQR 1481-1670); female offspring, 1448 (IQR 1271-1700) v. 1568 (IQR 1475-1744) mmHg of $\mathrm{CD} v$. SD, respectively).

Without any obvious programmed alteration to tonic endothelial (nitric oxide) activity or cardiac function, we next tested whether male and female offspring were rendered differentially reactive to the same inducing dietary stimulus in their mothers.

\section{Stimulated cardiovascular responses: salt sensitivity}

There was little measurable effect of high salt intake on cardiovascular status in the male and female offspring of all dietary groups. Circadian analyses did indicate, however, that with salt 

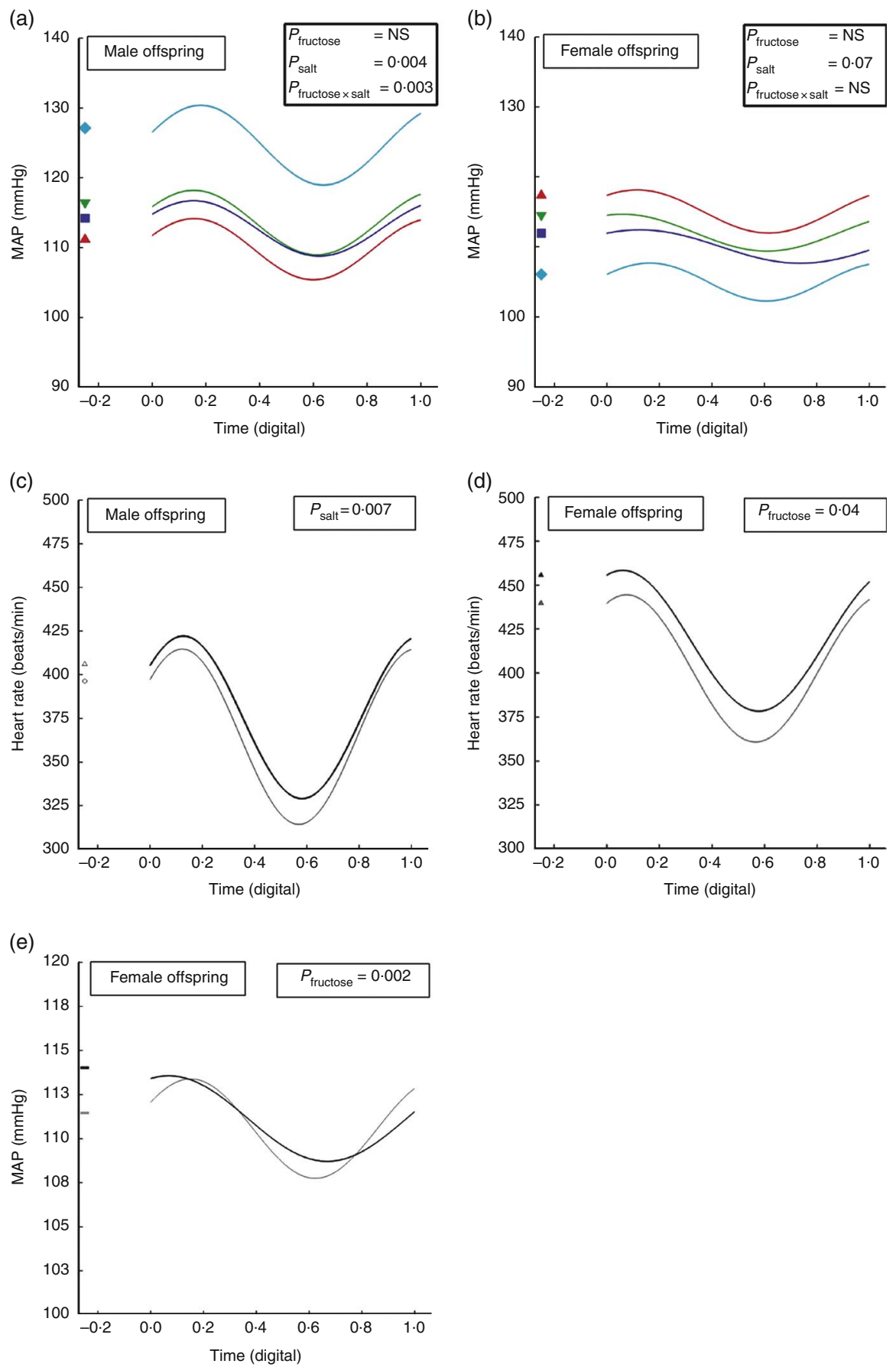

Fig. 1. Circadian analyses of pressure and heart rate in adult male and female offspring from dams fed fructose or salt. Circadian variation in mean arterial pressure (MAP; $a, b$ and e) and heart rate (c, d) derived from Fourier curves in adult male and female offspring of dams fed (1) control diet and water ad libitum (CD, $n 6$ male/ female offspring), (2) CD and $10 \%$ fructose in water ad libitum (fructose diet (FD), $n 5$ male/female offspring), (3) $4 \%$ salt diet (SD) and water ad libitum (SD, $n 5$ male/ female offspring) and (4) $4 \%$ SD and $10 \%$ fructose in water ad libitum (fructose/salt diet (FSD), $n 5$ male/female offspring). Fourier plots represent predicted mean regression curve for each group (GenStat version 16; VSNi Ltd). Digital time is $00.00=0.0000$ and $23 \mathrm{~h} 59 \mathrm{~min} 59 \mathrm{~s}=0.9999$. FSD, fructose/salt diet. $\wedge, \mathrm{SD} ; \nabla$, FD; $\square$ FSD; $\Delta, C D ; \triangle$, +ve salt; $\bigcirc-$ ve salt; $\_$, +ve fructose; $\_$, - ve fructose; $\mathbf{\Lambda}$, +ve fructose; $\boldsymbol{\Lambda},-$ ve fructose.

loading the offspring of fructose-exposed dams exhibited significantly blunted nocturnal dipping of pressure $(\beta$-coefficient male offspring; $3.9 v .5 .3($ sem 0.4$) \mathrm{mmHg} ; F=3.8 ; P_{\text {light } \times \text { salt }}=$ $0.001)$ and heart rate ( $\beta$-coefficient male offspring, $39.5 v .45 \cdot 3$ (sEM 2.1) $\mathrm{mmHg} ; F=6.3 ; P_{\text {light } \times \text { fructose }}=0.001$; female offspring, $37 \cdot 1$ v. $41.9($ SEM 1.7$\left.) \mathrm{mmHg} ; F=2 \cdot 5 ; P_{\text {light } \times \text { fructose }}=0 \cdot 01\right)$.

\section{Stimulated cardiovascular responses: fructose sensitivity}

Consumption of fructose per se had little cardiovascular effect in control offspring (CD effect size, 1.0 (SEM 2.3) $\mathrm{mmHg}$ ). In male offspring from salt-loaded dams, high fructose intake elicited a significant pressor response (SD effect size, 6 (SEM 2.6) $\mathrm{mmHg}$; 
Males

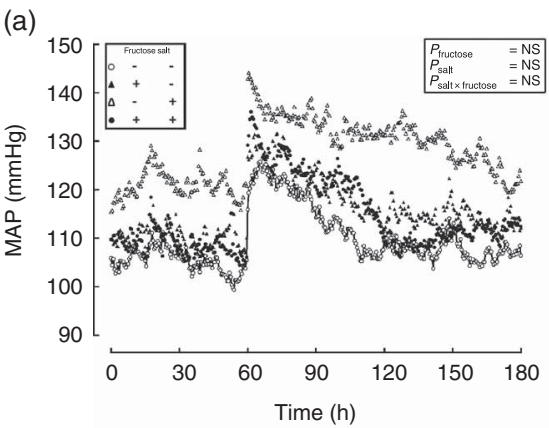

(c)

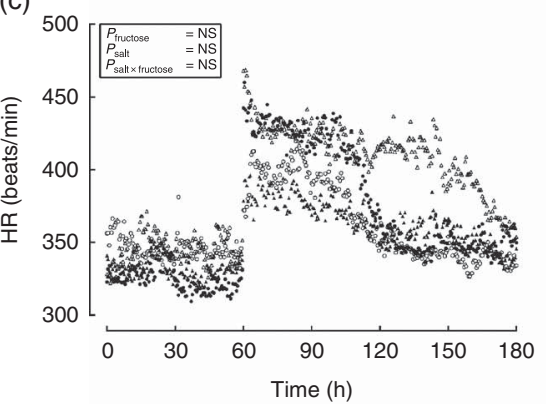

(e)

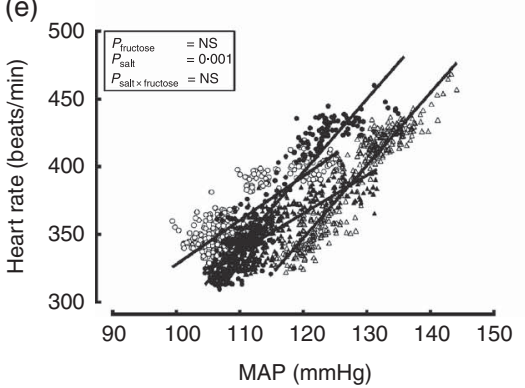

Females

(b)

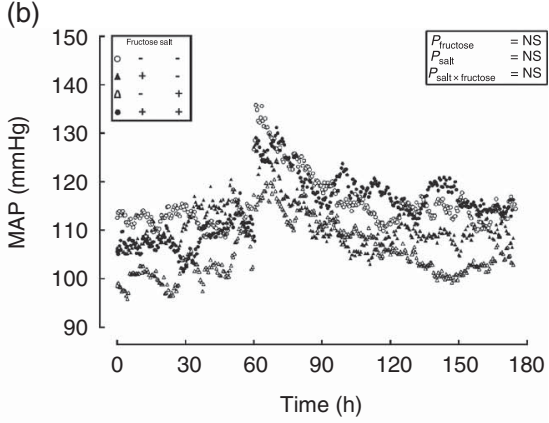

(d)

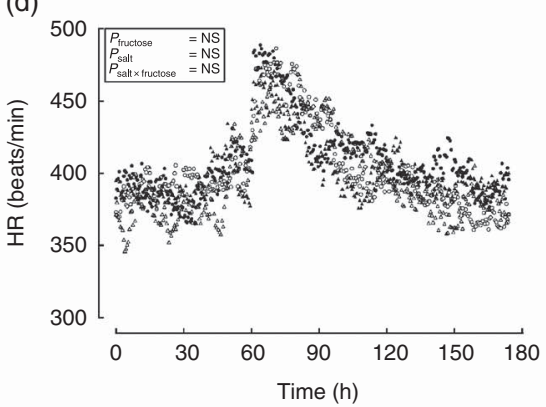

(f)

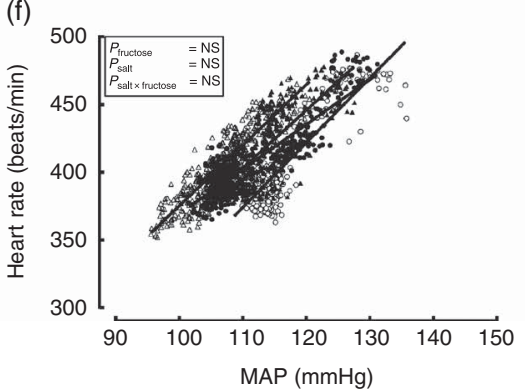

Fig. 2. Mean arterial pressure (MAP) $(a, b)$, heart rate (c, d) and slopes of the relationship (e, f) between mean arterial pressure and heart rate in male and female offspring at approximately 10 weeks of age from dams fed fructose and/or salt. Data are (O) control diet (CD) and water ad libitum $(n 6),(\mathbf{\Lambda}) \mathrm{CD}$ and $10 \%$ fructose in water ad libitum $(n 5),(\Delta) 4 \%$ salt diet (SD) and water ad libitum $(n 5),(\bullet) 4 \%$ SD and $10 \%$ fructose in water ad libitum $(n 5)$ for male and female offspring. Data were measured continuously (i.e. sampled at 2 outputs per $\mathrm{min}$ ) by telemetry for a 1-h baseline period and subsequently for $2 \mathrm{~h}$ after removal of their sibling from the cage. Regression lines were generated in Graphpad Prism 5.0.

$P=0 \cdot 002)$, which was greater in male offspring from fructoseloaded dams (FD effect size, 8.1 (SEM 2.6) mmHg; Fig. 4(a)). For female offspring, high fructose intake increased pulse pressure (effect size, $+5 \cdot 2$ (SEM 3.1) $\mathrm{mmHg} ; P=0 \cdot 005$ ), but this effect was 2-fold greater if their dams had also been fructose-loaded (FD effect size, 10.3 (SEM $3.1 \mathrm{mmHg}$; Fig. 4(b)). Heart rate varied with the light/dark cycle, as in the unchallenged state, but it was not overly influenced by 5 -d fructose consumption (Fig. 4(c) and (d)). In male rats, which were previously exposed to maternal salt loading, the increase in heart rate from day to night as the rats became active was diminished (a change of $54 v$. 60 beats/min \pm 5 ; $\left.P_{\text {salt }}<0.005\right)$.

\section{Heart rate variability}

During all challenges, heart rate variability (HRV) was calculated. HRV exhibited marked circadian and ultradian patterns under control conditions, which was unaffected by L-NAME treatment, salt loading or high intake of fructose (Fig. 5(a)-(f)). However, notably, regardless of the challenge, HRV distinctly peaked at 20.00 hours in all groups (Fig. 5(a)-(f)).

\section{Discussion}

The adverse metabolic consequences of increased consumption of extrinsic sugars, in particular fructose, has been widely reported $^{(33-36)}$. Only one study in mice ${ }^{(37)}$ and one in rats ${ }^{(38)}$ have described the cardiovascular effects of additional dietary salt on cardiovascular function; none has considered their interaction when fed to pregnant dams and, subsequently, to their offspring. In the current study, we reveal some clear circadian and sex-specific effects of high maternal intake of salt or fructose on cardiovascular physiology in the adult offspring. 


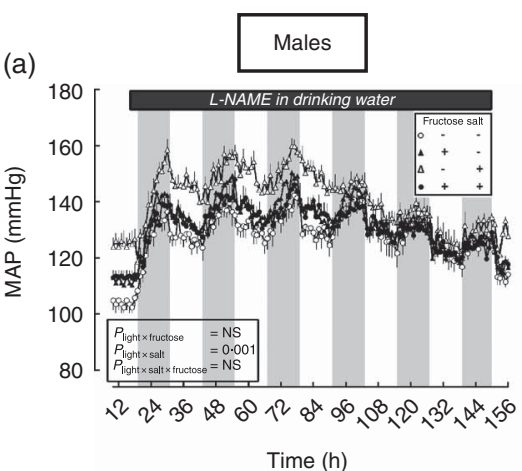

(c)
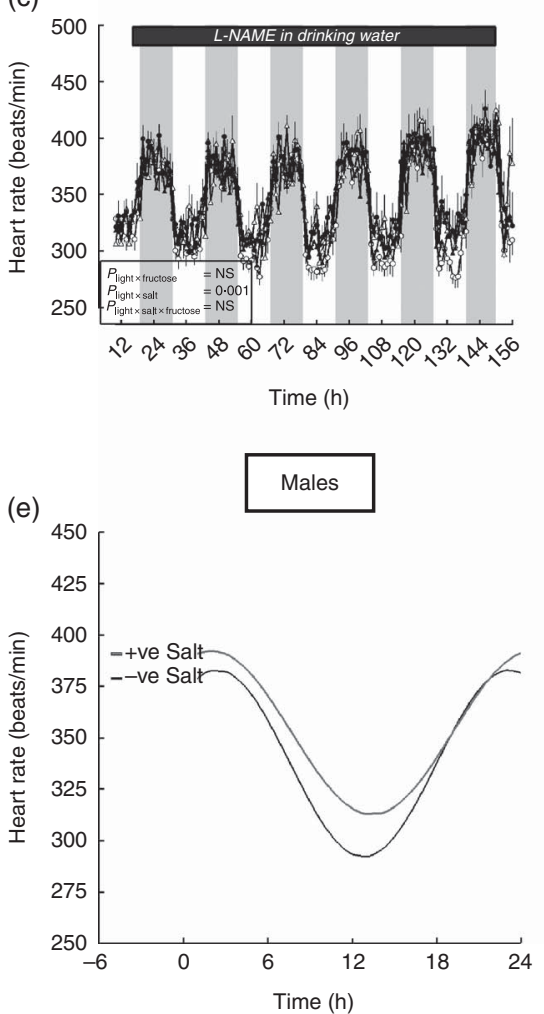

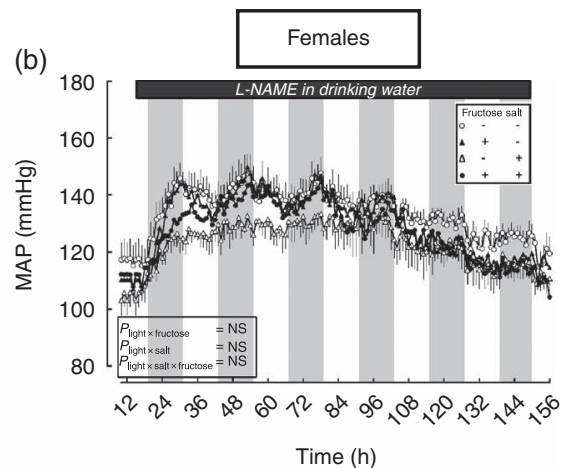

(d)
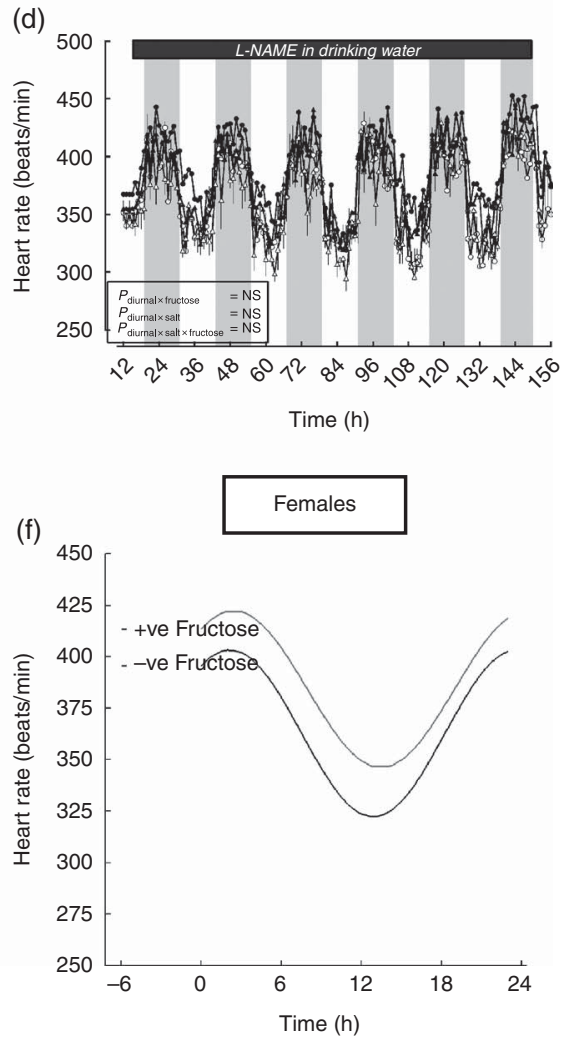

Fig. 3. Mean arterial pressure (MAP; a, b), heart rate (c, d) and Fourier curves (e, f) for circadian variation in heart rate in response to $N^{\mathrm{G}}$-nitro-L-arginine methyl ester (L-NAME) in the male and female offspring of dams fed fructose and/or salt. Data are (O) control diet (CD) and water ad libitum $(n 6),(\mathbf{\Delta}) \mathrm{CD}$ and $10 \%$ fructose in water ad libitum $(n 5),(\Delta) 4 \%$ salt diet (SD) and water ad libitum ( $n 5),(\bullet) 4 \%$ SD and $10 \%$ fructose in water ad libitum $(n 5)$ for male and female offspring. Data were measured intermittently (for $30 \mathrm{~s}$ every $15 \mathrm{~min}$ for $7 \mathrm{~d}$ ) by telemetry, and hourly means were calculated as a summary measure of the cardiovascular response. Data

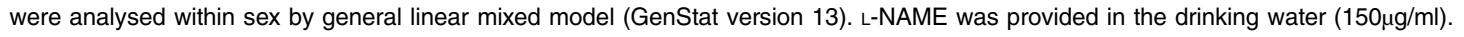

Two independent sex-specific phenotypes emerge that are retained despite no significant consumption of salt or fructose postnatally; maternal salt loading has distinct and marked hypertensive effects on male offspring, and maternal fructose loading appears to have greater cardiovascular effects on female offspring. Importantly, for fructose in particular, these effects in the female offspring are exacerbated by further fructose intake, as would naturally occur in human populations.

The adverse cardiovascular effects of increased consumption of salt have long been recognised ${ }^{(39)}$; for fructose, the deleterious consequences are the subject of much recent debate ${ }^{(40)}$. Taking an evidence-based approach, however, would favour the hypothesis that increased consumption of fructose after the introduction of high fructose maize syrup and sugar-sweetened beverages has had a negative impact on cardiovascular health ${ }^{(3,41)}$. When considering the impact of diet on health (including offspring health), the relativity is all important; early hominids evolved eating approximately $0.25 \mathrm{~g} / \mathrm{d}$ salt and no more than $2 \%$ energy/d from simple sugars. The current estimated average consumption is $8-12 \mathrm{~g} / \mathrm{d}$ salt and $18-25 \%$ energy/d from simple sugars. Relative to our ancestral diet, during which our physiology was moulded over many thousands of years, the current average diet represents a considerable physiological burden. In the context of developmental programming, in which maternal malnutrition may influence fetal development to result in adaptations that become 
(a)

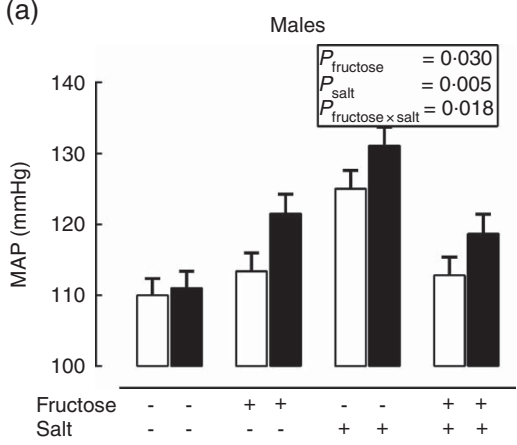

(c)

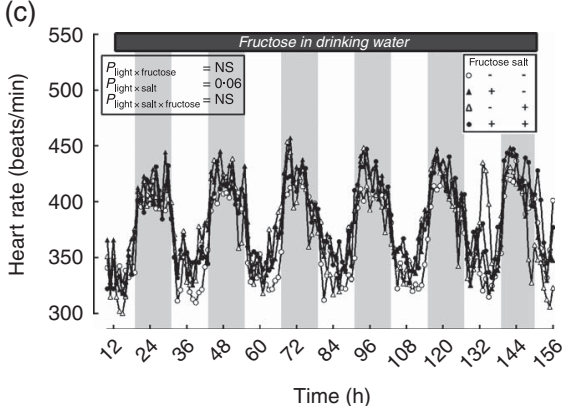

(b)

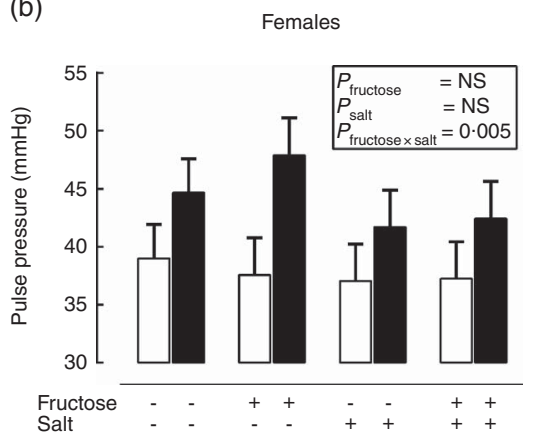

(d)

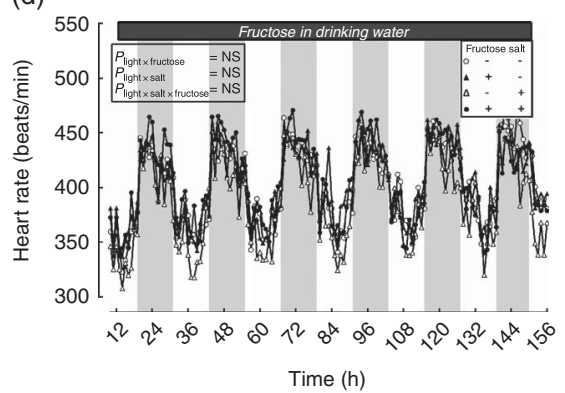

Fig. 4. Mean arterial pressure (MAP) (a), pulse pressure (b) and summary measures of heart rate (c, d) during fructose ingestion in the male and female offspring of

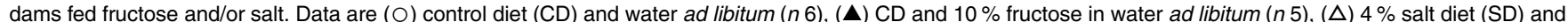
water ad libitum $(n 5),(\bullet) 4 \%$ SD and $10 \%$ fructose in water ad libitum $(n 5)$ for male and female offspring. Data were measured intermittently (for 30 s every 15 min for $7 \mathrm{~d})$ by telemetry and hourly means were calculated as a summary measure of the cardiovascular response. Data were analysed within sex by general linear mixed model (GenStat version 13). Fructose was provided in the drinking water (10\% solution). $\square$, Water; $\square, 10 \%$ fructose.

deleterious in a westernised nutritional environment, it is unsurprising that such a physiological burden is not without effect. Using an animal model to recapitulate a westernised dietary pattern, the current study illustrates how this burden may translate to the offspring, and how these responses are sexand nutrient-specific. For salt-loaded dams, effect size in sibling offspring is approximately $25 \mathrm{mmHg}$ (male offspring are hypertensive (approximately $15 \mathrm{mmHg}$ above controls), and female offspring hypotensive (approximately $10 \mathrm{mmHg}$ below controls)). Such large sex-specific effect sizes are rarely observed $^{(42,43)}$.

Sex-specific effects are often observed within the developmental programming paradigm ${ }^{(44)}$ but, to our knowledge, none as marked as in the current study. This study was designed to illustrate potential sex-specific, delayed developmental effects but not to interrogate potential mechanisms should they arise. For example, whilst a number of models have inferred sexspecific effects of programming by adopting the relatively crude approach of gonad removal, a more appropriate intervention would be to use highly specific and reversible sex-hormone antagonists longitudinally. Some excellent recent studies that have shown programming of a sex-specific cardiovascular phenotype (such as increased blood pressure in male but not female offspring) have identified an absence of oestrogen in male offspring as a causal factor ${ }^{(42,43)}$; in effect, oestrogen acts as a 'pro-survival factor' mitigating (perhaps epigenetically) the adverse consequences of a nutritionally poor developmental environment until concentrations decline in middle-age and morbidity and mortality rates (e.g. for cardiovascular outcomes) in female offspring begin to rise - the basis for oestrogen replacement therapy ${ }^{(44)}$. However, being genetically male or female and interacting differently with the immediate (e.g. intrauterine) environment could be important; for example, periconceptional exposure to a maternal methyl-deficient diet for only $6 \mathrm{~d}$ (day 0-6 gestation) revealed significant sex-specific differential DNA methylation of CPG islands in the fetal livers at day 90 of gestation - i.e. of the altered loci as a result of the dietary treatment, $53 \%$ were specific to male and only $12 \%$ were specific to female ${ }^{(15)}$.

Programmed alterations of cardiovascular control in saltexposed offspring appears independent of tonic endothelial nitric oxide; if this were the case, then L-NAME treatment should have revealed differences in short-term responses (i.e. the magnitude of increase in first 8-12 h) or long-term regulation. However, a simple procedure to induce temporal anxiety removing the cage mate for a 24-h period - does reveal marked differences in male, salt-exposed offspring. This has two important consequences: first, the generation of curves of the coupling between pressure and heart rate at this time indicates that salt-exposed hypertensive male offspring, but not nonhypertensive female siblings, have a greater rate of rise of heart rate per unit pressure relative to female salt-exposed offspring. This suggests a centrally mediated alteration at the level of the brain or peripheral autonomic nervous system and/or an effect on cardiac function. The latter can be ruled out, as ex vivo cardiac function, as shown by the Langendorff preparation, was not significantly different. Furthermore, we have previously shown that the offspring of salt-loaded dams have altered 

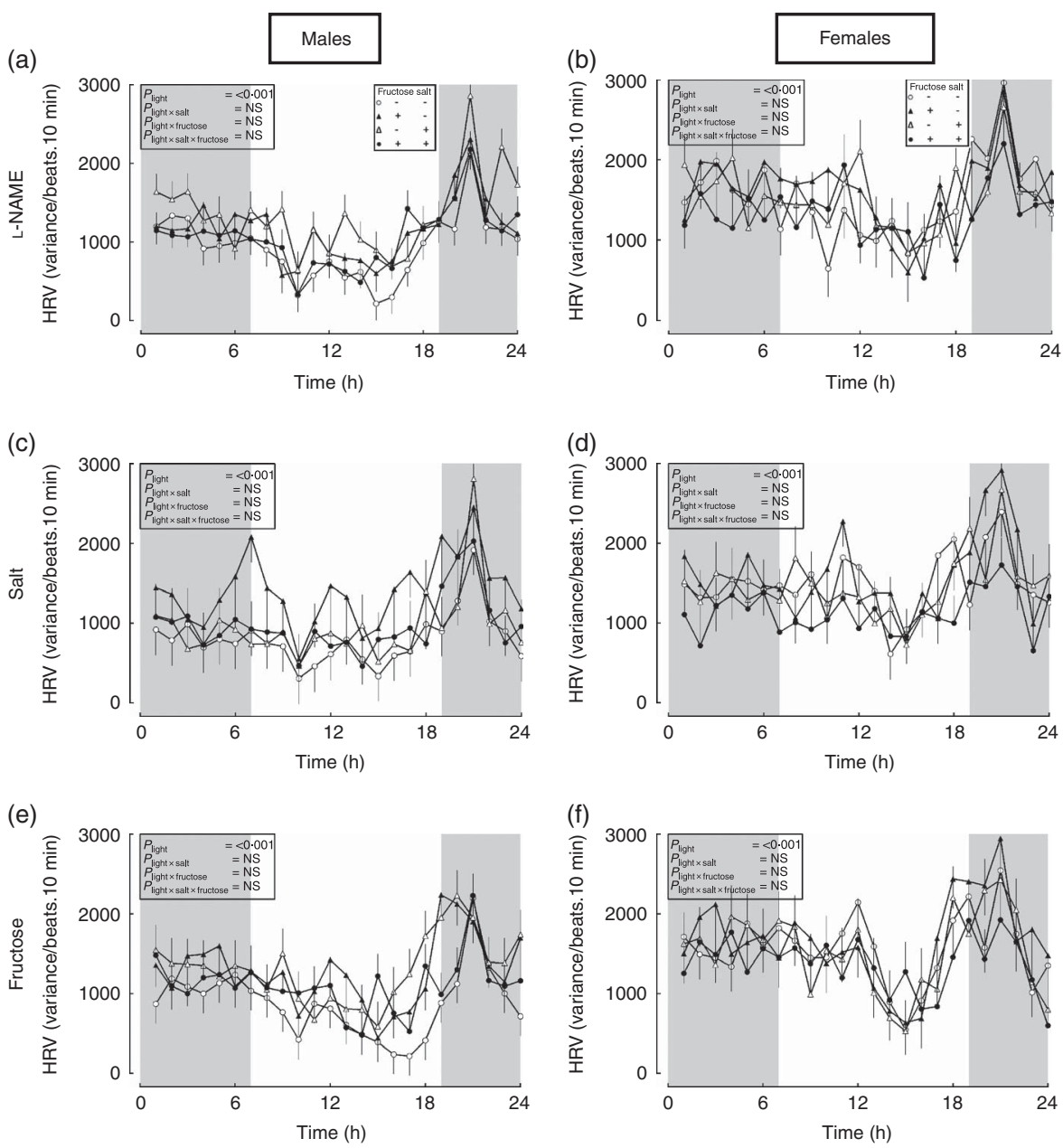

Fig. 5. Heart rate variability (HRV) in male and female offspring from dams fed $(O)$ control diet $(C D)$ and water ad libitum $(n 6),(\mathbf{\Delta}) \mathrm{CD}$ and $10 \%$ fructose in water ad libitum $(n 5),(\Delta) 4 \%$ salt diet (SD) and water ad libitum $(n 5),(\bullet) 4 \%$ SD and $10 \%$ fructose in water ad libitum $(n 5)$ for male and female offspring during $5 \mathrm{~d}$ of (a, b) $N^{G}$-nitro-L-arginine methyl ester (L-NAME) treatment, (c, d) $4 \%$ salt loading and (e, f) $10 \%$ fructose in drinking water. Heart rate was derived from the radiotelemetric pressure pulse and recorded intermittently (for $30 \mathrm{~s}$ every $15 \mathrm{~min}$ ) for the duration $(7 \mathrm{~d})$ of each nutritional challenge. HRV was calculated as the variance $\left(\mathrm{SD}^{2}\right)$ in heart rate for each hour of recording. Data were highly positively skewed and were therefore analysed by general linear mixed model with a gamma error distribution and logarithm-link function; back-transformed predicted means are presented (GenStat version 16).

set-points for osmolar regulation - a phenotype indicative of alterations at the level of the brain ${ }^{(24)}$. In addition, the data clearly indicate that measurements of resting blood pressure in telemetered rats should always be conducted with same-sex sibling cage mates in order to achieve a true 'resting or ambulatory reading'; single-housed rats are easily stressed, which has a marked negative impact on resting cardiovascular variables.

For the first time, we provide evidence that increased maternal fructose consumption has important effects on adult offspring cardiovascular control. Resting blood pressure was unaltered by increased maternal fructose intake, but the circadian oscillation in pressure and heart rate was significantly blunted, reflective of a 'non-dipping' nocturnal pattern - previously identified as a significant risk factor for later $\mathrm{CVD}^{(45)}$. This finding is intriguing considering the limited exposure to fructose; none had consumed any fructose, as they were weaned at 3 weeks of age. A number of studies have previously reported a pressor effect of fructose either given acutely, using high doses (66\% of total energy intake ${ }^{(46)}$ ), or chronically (using lower doses ${ }^{(35)}$ ) and others reporting no effects ${ }^{(47)}$. Furthermore, our data suggest that maternal diet renders offspring (in particular female offspring) with a residual, increased sensitivity to further fructose intake. Mean arterial or pulse pressure in male and female offspring increased significantly more in prenatally fructose-exposed groups relative to control animals. The fact that chronic L-NAME treatment did not reveal any difference in fructose-exposed groups suggests no residual involvement of tonic nitric oxide activity. A recent study demonstrated that an altered pattern of vascular smooth muscle prostanoid release may be a contributing factor to fructoseinduced vascular sensitivity ${ }^{(48)}$, but equally up-regulation of other vasoconstrictor, anti-natriuretic or diminished vasodilatory pathways may be causal. We have measured a number of fructose-induced advanced glycation end products such as fructosamine (an indicator of fructose-induced protein glycosylation), uric acid and glucose and found no difference in the basal state to account for alterations in fructose sensitivity. 
Acute fructose ingestion has been shown to increase blood pressure, likely through an effect on cardiac sympathetic sensitivity $^{(49)}$. The current study illustrates that the effects of fructose ingestion after being exposed in utero to a maternal diet high in fructose have a distinct sex-specific bias, with female offspring being more fructose-sensitive.

Finally, the current study clearly illustrates that moderate over-consumption of salt and/or fructose by dams during pregnancy and lactation is able, in the offspring, to recapitulate many of the known pathophysiological effects of these micronutrients despite little exposure of the offspring to these diets. This has marked implication for NCD in Western populations. Continued intake of refined, low nutritional-quality diets in the next generation, following maternal over-consumption, has the potential to vertically transmit adverse health outcomes through generations. Reversal of this trend is going to require preventative action before birth, and as a result it will also take generations to effect a response. Given the implications for human populations, we would also strongly endorse recent commentaries and initiatives to reduce both the quantity of salt $^{(50)}$ and fructose ${ }^{(18)}$ consumed as part of the modern Western diet.

\section{Acknowledgements}

The authors express their gratitude for the help and support provided by the Bioscience Research Unit on the Sutton Bonington Campus and Julie March for performing the radiotelemetry transmitter implantations. All work in this manuscript was performed on the Sutton Bonington Campus, University of Nottingham.

This work was supported by a BBSRC doctoral training grant to C. G. (50:50) with The University of Nottingham. Grant support from The Nutricia Research Foundation and The School of Veterinary Medicine and Science is gratefully acknowledged.

D. S. G., C. G. and S. M. G. designed research; C. G. and D. S. G. conducted the research; S. M. G. provided essential materials; and D. S. G., C. G. and S. M. G. wrote and critically evaluated the paper. D. S. G. has primary responsibility for its final content.

The authors declare that they have no conflicts of interest.

\section{References}

1. Eaton SB, Konner MJ \& Cordain L (2010) Diet-dependent acid load, Paleolithic nutrition, and evolutionary health promotion. Am J Clin Nutr 91, 295-297.

2. Cordain L, Eaton SB, Sebastian A, et al. (2005) Origins and evolution of the Western diet: health implications for the 21st century. Am J Clin Nutr 81, 341-354.

3. Strohle A, Hahn A \& Sebastian A (2010) Latitude, local ecology, and hunter-gatherer dietary acid load: implications from evolutionary ecology. Am J Clin Nutr 92, 940-945.

4. Popkin BM (2006) Global nutrition dynamics: the world is shifting rapidly toward a diet linked with noncommunicable diseases. Am J Clin Nutr 84, 289-298.

5. Daar AS, Singer PA, Persad DL, et al. (2007) Grand challenges in chronic non-communicable diseases. Nature 450, 494-496.

6. World Health Organization (2005) Preventing Chronic Diseases: A Vital Investment. Geneva: WHO.
7. Narayan KMV, Ali MK \& Koplan JP (2010) Global noncommunicable diseases - where worlds meet. New Engl J Med 363, 1196-1198.

8. Lindeberg S, Jonsson T, Granfeldt Y, et al. (2007) A Palaeolithic diet improves glucose tolerance more than a Mediterranean-like diet in individuals with ischaemic heart disease. Diabetologia 50, 1795-1807.

9. Bibbins-Domingo K, Chertow GM, Coxson PG, et al. (2010) Projected effect of dietary salt reductions on future cardiovascular disease. New Engl J Med 362, 590-599.

10. Chen L, Caballero B, Mitchell DC, et al. (2010) Reducing consumption of sugar-sweetened beverages is associated with reduced blood pressure: a prospective study among United States adults. Circulation 121, 2398-2406.

11. Crispi F, Bijnens B, Figueras F, et al. (2010) Fetal growth restriction results in remodeled and less efficient hearts in children. Circulation 121, 2427-2436.

12. Franks PW, Hanson RL, Knowler WC, et al. (2010) Childhood obesity, other cardiovascular risk factors, and premature death. New Engl J Med 362, 485-493.

13. Whincup PH, Kaye SJ, Owen CG, et al. (2008) Birth weight and risk of type 2 diabetes: a systematic review. JAMA $\mathbf{3 0 0}$, 2886-2897.

14. Langley-Evans SC, Phillips GJ \& Jackson AA (1994) In utero exposure to maternal low protein diets induces hypertension in weanling rats, independently of maternal blood pressure changes. Clin Nutr 13, 319-324.

15. Khan IY, Dekou V, Douglas G, et al. (2005) A high-fat diet during rat pregnancy or suckling induces cardiovascular dysfunction in adult offspring. Am J Physiol Regul Integr Comp Physiol 288, R127-R133.

16. Sedova L, Seda O, Kazdova L, et al. (2007) Sucrose feeding during pregnancy and lactation elicits distinct metabolic response in offspring of an inbred genetic model of metabolic syndrome. Am J Physiol Endocrinol Metab 292, E1318-E1324.

17. National Food Survey (2007) Household food expenditure and consumption and nutrient intake 1974-2007.

18. Johnson RK, Appel LJ, Brands M, et al. (2009) Dietary sugars intake and cardiovascular health: a scientific statement from the American Heart Association. Circulation 120, 1011-1020.

19. Bernstein AM \& Willett WC (2010) Trends in 24-h urinary sodium excretion in the United States, 1957-2003: a systematic review. Am J Clin Nutr 92, 1172-1180.

20. Alzamendi A, Castrogiovanni D, Gaillard RC, et al. (2010) Increased male offspring's risk of metabolic-neuroendocrine dysfunction and overweight after fructose-rich diet intake by the lactating mother. Endocrinology 151, 4214-4223.

21. Contreras RJ, Wong DL, Henderson R, et al. (2000) High dietary $\mathrm{NaCl}$ early in development enhances mean arterial pressure of adult rats. Physiol Behav 71, 173-181.

22. Porter JP, King SH \& Honeycutt AD (2007) Prenatal high-salt diet in the Sprague-Dawley rat programs blood pressure and heart rate hyperresponsiveness to stress in adult female offspring. Am J Physiol Regul Integr Comp Physiol 293, R334-R342.

23. Soria A, Chicco A, Mocchiutti N, et al. (1996) A sucrose-rich diet affects triglyceride metabolism differently in pregnant and nonpregnant rats and has negative effects on fetal growth. J Nutr 126, 2481-2486.

24. Gray C, Al-Dujaili EA, Sparrow AJ, et al. (2013) Excess maternal salt intake produces sex-specific hypertension in offspring: putative roles for kidney and gastrointestinal sodium handling. PLOS ONE 8, e72682.

25. He FJ, Marrero NM \& MacGregor GA (2008) Salt intake is related to soft drink consumption in children and adolescents: a link to obesity? Hypertension 51, 629-634. 
26. Ojeda NB, Grigore D \& Alexander BT (2008) Developmental programming of hypertension: insight from animal models of nutritional manipulation. Hypertension 52, 44-50.

27. Duma D, Collins JB, Chou JW, et al. (2010) Sexually dimorphic actions of glucocorticoids provide a link to inflammatory diseases with gender differences in prevalence. Sci Signal 3, ra74.

28. Zucker I \& Beery AK (2010) Males still dominate animal studies. Nature 465, 690-690.

29. Kim AM, Tingen CM \& Woodruff TK (2010) Sex bias in trials and treatment must end. Nature 465, 688-689.

30. Gray C, Long S, Green C, et al. (2013) Maternal fructose and/ or salt intake and reproductive outcome in the rat: effects on growth, fertility, sex ratio, and birth order. Biol Reprod 89, 51.

31. Kramer K \& Kinter LB (2003) Evaluation and applications of radiotelemetry in small laboratory animals. Physiol Genomics 13, 197-205.

32. National Research Council (US) Subcommittee on Laboratory Animal Nutrition (1995) Nutrient Requirements of Laboratory Animals, 4th Ed. Washington, DC: National Academy of Sciences.

33. Tappy L \& Le K-A (2010) Metabolic effects of fructose and the worldwide increase in obesity. Physiol Rev 90, 23-46.

34. Kanerva N, Sandboge S, Kaartinen NE, et al. (2014) Higher fructose intake is inversely associated with risk of nonalcoholic fatty liver disease in older Finnish adults. Am J Clin Nutr 100, 1133-1138.

35. Jalal DI, Smits G, Johnson RJ, et al. (2010) Increased fructose associates with elevated blood pressure. J Am Soc Nephrol 21, 1543-1549.

36. Stanhope KL, Schwarz JM, Keim NL, et al. (2009) Consuming fructose-sweetened, not glucose-sweetened, beverages increases visceral adiposity and lipids and decreases insulin sensitivity in overweight/obese humans. J Clin Invest 119 , 1322-1334.

37. Huang DY, Boini KM, Friedrich B, et al. (2006) Blunted hypertensive effect of combined fructose and high-salt diet in gene-targeted mice lacking functional serum- and glucocorticoid-inducible kinase SGK1. Am J Physiol Regul Integr Comp Physiol 290, R935-R944.

38. Song J, Hu X, Shi M, et al. (2004) Effects of dietary fat, $\mathrm{NaCl}$, and fructose on renal sodium and water transporter abundances and systemic blood pressure. Am J Physiol Renal Physiol 287, F1204-F1212.

39. Bang HO, Bechgaard P \& Nielsen AL (1949) Low-salt diet in treatment of hypertension and hypertensive heart disease. $\mathrm{Br}$ Med J 2, 1203-1206.

40. Johnson RJ, Perez-Pozo SE, Sautin YY, et al. (2009) Hypothesis: could excessive fructose intake and uric acid cause type 2 diabetes? Endocr Rev 30, 96-116.

41. Fung TT, Malik V, Rexrode KM, et al. (2009) Sweetened beverage consumption and risk of coronary heart disease in women. Am J Clin Nutr 89, 1037-1042.

42. Sinclair KD, Allegrucci C, Singh R, et al. (2007) DNA methylation, insulin resistance, and blood pressure in offspring determined by maternal periconceptional $\mathrm{B}$ vitamin and methionine status. Proc Natl Acad Sci U S A 104, 1935119356.

43. Grigore D, Ojeda NB \& Alexander BT (2008) Sex differences in the fetal programming of hypertension. Gend Med 5, Suppl A, S121-S132.

44. Aiken CE \& Ozanne SE (2013) Sex differences in developmental programming models. Reproduction 145, R1-R13.

45. de la Sierra A, Redon J, Banegas JR, et al. (2009) Prevalence and factors associated with circadian blood pressure patterns in hypertensive patients. Hypertension 53, 466-472.

46. Hwang I, Ho H, Hoffman B, et al. (1987) Fructose-induced insulin resistance and hypertension in rats. Hypertension $\mathbf{1 0}$, 512-516.

47. D'Angelo G, Elmarakby AA, Pollock DM, et al. (2005) Fructose feeding increases insulin resistance but not blood pressure in Sprague-Dawley rats. Hypertension 46, 806-811.

48. Puyo AM, Zabalza M, Mayer M, et al. (2009) Time course of vascular prostanoid production in the fructosehypertensive rat. Auton Autacoid Pharmacol 29, 135-139.

49. Brown CM, Dulloo AG, Yepuri G, et al. (2008) Fructose ingestion acutely elevates blood pressure in healthy young humans. Am J Physiol Regul Integr Comp Physiol 294, R730-R737.

50. He FJ, Jenner KH \& MacGregor GA (2010) WASH-world action on salt and health. Kidney Int 78, 745-753. 